\title{
Cytogenetic and molecular identification of a wheat-Leymus mollis alien multiple substitution line from octoploid Tritileymus x Triticum durum
}

\author{
Y.H. Pang ${ }^{1}$, J.X. Zhao ${ }^{1}$, W.L. Du ${ }^{1}$, Y.L. Li ${ }^{1}$, J. Wang ${ }^{1}$, L.M. Wang ${ }^{1}$, J. Wu ${ }^{1}$, \\ X.N. Cheng ${ }^{2}$, Q.H. Yang ${ }^{1}$ and X.H. Chen ${ }^{1}$ \\ ${ }^{1}$ Key Laboratory of Plant Genetics and Breeding Engineering of Shaanxi Province, \\ College of Agronomy, Northwest A\&F University, Yangling, Shaanxi, China \\ ${ }^{2}$ College of Life Sciences, Northwest A\&F University, Yangling, Shaanxi, China \\ Corresponding author: X.H. Chen \\ E-mail:cxh2105@yahoo.com.cn
}

Genet. Mol. Res. 13 (2): 3903-3913 (2014)

Received January 30, 2013

Accepted July 4, 2013

Published May 23, 2014

DOI http://dx.doi.org/10.4238/2014.May.23.1

\begin{abstract}
Leymus mollis (Trin.) Pilger (NsNsXmXm, $2 \mathrm{n}=28$ ), a wild relative of common wheat, possesses many traits that are potentially valuable for wheat improvement. In order to exploit and utilize the useful genes of L. mollis, we developed a multiple alien substitution line, 10DM50, from the progenies of octoploid Tritileymus M842-16 x Triticum durum cv. D4286. Genomic in situ hybridization analysis of mitosis and meiosis (metaphase I), using labeled total DNA of Psathyrostachys huashanica as probe, showed that the substitution line 10DM50 was a cytogenetically stable alien substitution line with 36 chromosomes from wheat and three pairs of Ns genome chromosomes from L. mollis. Simple sequence repeat analysis showed that the chromosomes $3 \mathrm{D}, 6 \mathrm{D}$, and $7 \mathrm{D}$ were absent in 10DM50. Expressed sequence tag-sequence tagged sites analysis showed that new chromatin from $3 \mathrm{Ns}, 6 \mathrm{Ns}$, and $7 \mathrm{Ns}$ of $L$. mollis were detected in 10DM50. We deduced that the substitution line 10DM50 was a multiple alien substitution line with the $3 \mathrm{D}, 6 \mathrm{D}$, and 7D chromosomes replaced by $3 \mathrm{Ns}$, $6 \mathrm{Ns}$, and $7 \mathrm{Ns}$ from $L$. mollis. 10DM50 showed high resistance
\end{abstract}


to leaf rust and significantly improved spike length, spikes per plant, and kernels per spike, which are correlated with higher wheat yield. These results suggest that line 10DM50 could be used as intermediate material for transferring desirable traits from L. mollis into common wheat in breeding programs.

Key words: Alien substitution line; Leaf rust; Leymus mollis; Molecular cytogenetics; Triticum aestivum

\section{INTRODUCTION}

Wild relatives of common wheat (Triticum aestivum L.), which are grown in extreme environmental conditions, have interested wheat breeders as a tremendous genetic pool of beneficial traits that can be transferred to cultivated species in common wheat breeding programs (Baum et al., 1992; Jiang et al., 1994; Kishii et al., 2004; Lin et al., 2007). Leymus mollis (Trin.) Pilger (NsNsXmXm, $2 \mathrm{n}=28$ ), a distant relative species of wheat, with a Ns genome originating from the genus Psathyrostachys and an Xm genome of unknown origin, has been identified to have many potentially valuable traits for wheat improvement, such as tolerance to drought and low temperatures, resistance to various diseases including scab, powdery mildew, stripe rust, and yellow dwarf virus, and exceptionally large spikes and vigorous growth (Wang and Jensen, 1994; Kishii et al., 2003; Zhou et al., 2010). With the aim of introducing useful genes to common wheat, wide hybridizations between L. mollis and common wheat have been independently carried out in Russia (Tsitsin, 1965), China (Fu et al., 1993), Iceland (AnamthawatJónsson et al., 1997), and Sweden (Merker and Lantai, 1998), resulting in the development of a series of valuable intermediate materials.

Hybridization was successfully performed between common wheat 7182 (AABBDD, $2 \mathrm{n}=42$ ) and L. mollis (NsNsXmXm, $2 \mathrm{n}=28$ ) in our research group, and the hybrids were obtained by embryo rescue and colchicine treatment in the 1980s (Chen et al., 1985). Different types of Tritileymus, octoploid amphiploids, and assorted derivatives were obtained from backcrossed and intercrossed progenies (Fu et al., 1996). Cytogenetic analysis of octoploid Tritileymus M842 indicated that the lines M842-4, M842-8, M842-12, and M842-16 had the karyotype of AABBDDNsNs, while the line M842-13 was AABBDDXmXm (Wang et al., 2000). A multiple alien substitution line, 05DM6, carrying three pairs of Ns chromosomes was selected from the progenies of octoploid Tritileymus M842-12 and Triticum durum cv. Trs-372 (Zhao et al., 2012).

Octoploid Tritileymus M842-16 (AABBDDNsNs, 2n = 56) still possesses many interesting agronomic characters from L. mollis, such as cold and drought tolerance, and resistance to stripe rust, stem rust, scab, and powdery mildew (Jing et al., 2001). In order to further exploit and utilize the valuable germplasm material, the hybridization between octoploid Tritileymus M842-16 and Triticum durum cv. D4286 was made in 2007. After five generations of selfing, accompanied by cytogenetic analysis and artificial selection, an alien substitution line, 10DM50, was selected. The objectives of the present study were: 1) to analyze the meiosis behaviors of 10DM50 using cytogenetic analysis, 2) to determine the chromosome composition of 10DM50 using molecular markers including simple sequence repeats (SSRs) and expressed sequence tag-sequence tagged sites (EST-STSs), and 3) to assess its potentially valuable agronomic traits for wheat improvement. 


\section{MATERIAL AND METHODS}

\section{Plant materials}

The plant materials used in this study were Leymus mollis (Trin.) Pilger (NsNsXmXm, $2 n=28)$ accession BM01, Psathyrostachys huashanica Keng (NsNs, $2 n=14)$ accession 0503383, octoploid Tritileymus M842-16 (AABBDDNsNs, $2 \mathrm{n}=56$ ), Triticum aestivum $\mathrm{L}$. cv. 7182 (AABBDD, $2 \mathrm{n}=42$ ), Triticum durum L. cv. D4286 (AABB, $2 \mathrm{n}=28$ ), and the wheat- $L$. mollis substitution line 10DM50, which are all deposited in the College of Agronomy at Northwest A\&F University. T. aestivum cv. 7182 and L. mollis were the parents of octoploid Tritileymus M842-16. The wheat-L. mollis alien substitution line, 10DM50, was obtained from the $\mathrm{F}_{5}$ progeny of the cross between $T$. durum cv. D4286 and octoploid Tritileymus M842-16. The total DNA of $P$. huashanica was used as a probe to detect the Ns chromatin of substitution line 10DM50.

\section{Observations of mitosis and meiosis}

Root tips from germinating seeds of the substitution line 10DM50 were pretreated in ice water for $24 \mathrm{~h}$, and fixed in Carnoy's fixative (ethanol:acetic acid $=3: 1, \mathrm{v} / \mathrm{v}$ ). Then, the fixed root tips were digested with $1 \%$ cellulose $+1 \%$ pectinase, squashed, and stained with aceto-carmine. Young spikes containing meiotic divisions in the pollen mother cells (PMC) of 10DM50 were collected and pretreated as described for root tips but without digestion. Observations and documentation were conducted under a microscope (Olympus BX60).

\section{Genomic in situ hybridization (GISH)}

The fixed root tip and anthers of young spikes were squashed in $45 \%$ acetic acid on slides. Cover slips were removed by freezing the slides in liquid nitrogen. Total genomic DNA of $P$. huashanica was extracted from fresh leaves by the CTAB (Cota-Sánchez et al., 2006) method and labeled with Dig-Nick-Translation Mix (Roche, Germany) following manufacturer instructions, which served as the hybridization probe. The $40 \mathrm{~mL}$ denatured hybridization mix

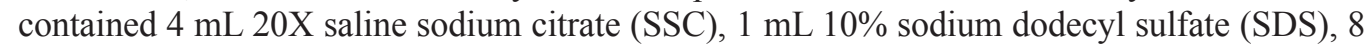

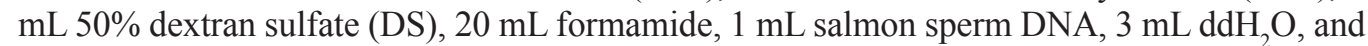
$3 \mathrm{~mL}$ labeled probe DNA. The GISH protocol was performed as described previously (Walling et al., 2005). Digoxigenin-labeled probes were visualized with anti-digoxigenin-FITC and the chromosomes were counterstained with propidium iodide. Images were captured with a CCD camera (Pixera Penguin 150CL) using a fluorescence microscope (Olympus BX60), and processed with Adobe Photoshop to adjust brightness and contrast when required.

\section{SSR and EST-STS marker analysis}

Genomic DNA was isolated from the fresh leaves of the line 10DM50, octoploid Tritileymus M842-16, Triticum durum D4286, common wheat 7182, and L. mollis by the CTAB method. Fourteen previously described pairs of D-genome-specific SSR primers were selected (Röder et al., 1998; Pestsova et al., 2000) and used to determine the D genome composition of 10DM50 (Table 1). EST-STS primer pairs showing polymorphic bands between octoploid Tritileymus and common wheat 7182 were selected from 140 EST-STS primer pairs from an online database 
(http://wheat.pw.usda.gov/SNP/new/pcr_primers.shtml), and used to detect the homology group attributes of the alien chromosomes in 10DM50 (Table 2). Polymerase chain reaction (PCR) amplifications were performed in a $20 \mu \mathrm{L}$ volume containing $1 \mathrm{X}$ PCR buffer, $1.5 \mathrm{mM} \mathrm{MgCl}, 200$ $\mu \mathrm{M}$ dNTP, $0.4 \mu \mathrm{M}$ each forward and reverse primers, $1 \mathrm{U}$ Taq polymerase, and $100 \mathrm{ng}$ template DNA. The amplification program was denaturing for $4 \mathrm{~min}$ at $94^{\circ} \mathrm{C}$, followed by 35 cycles of 45 $\mathrm{s}$ denaturing at $94^{\circ} \mathrm{C}, 45 \mathrm{~s}$ annealing at $50-60^{\circ} \mathrm{C}$ (depending on the specific primers), and $1 \mathrm{~min}$ extension at $72^{\circ} \mathrm{C}$, and then $10 \mathrm{~min}$ final extension at $72^{\circ} \mathrm{C}$. The PCR amplification products were separated on $8 \%$ non-denaturing polyacrylamide gels and were visualized by silver staining.

\section{Investigation of agronomic traits}

Random samples of 10DM50 and its parents, i.e., octoploid Tritileymus M842-16 and the durum wheat cv. D4286, as well as an earlier parent, common wheat cv. 7182, were investigated at the mature stage with respect to seven morphological traits: plant height, spikes per plant, spike length, spikelets per spike, kernels per spike, 1000-kernel weight, and selfing-fertility. At least 10 samples were analyzed for each trait. Variance analysis was used to evaluate these agronomic traits of 10DM50 and its parents, common wheat cv. 7182, durum wheat D4286, and octoploid Tritileymus M842. The adult plant response to leaf rust was assessed via natural infection with a highly susceptible wheat line sown next to the tested lines. The agronomic traits investigation and the evaluation of the adult stage resistance to leaf rust were assessed in field conditions.

\section{RESULTS}

\section{Cytogenetic analysis of 10DM50}

Mitotic observations of 20 randomly selected 10DM50 cells showed that all cells had the chromosome number $2 n=42$ (Figure 1A). The meiotic analysis of 40 PMCs at metaphase I showed that 38 cells had a chromosome configuration of $2 n=21 \mathrm{II}$, and two cells had $2 \mathrm{n}=$ 20 II +2 I (Figure 1B). No trivalents or quadrivalents were observed at metaphase I, and no chromosomes lagged at anaphase I. This indicated that the meiotic behavior of 10DM50 was regular, which suggested that it was a cytogenetically stable line.

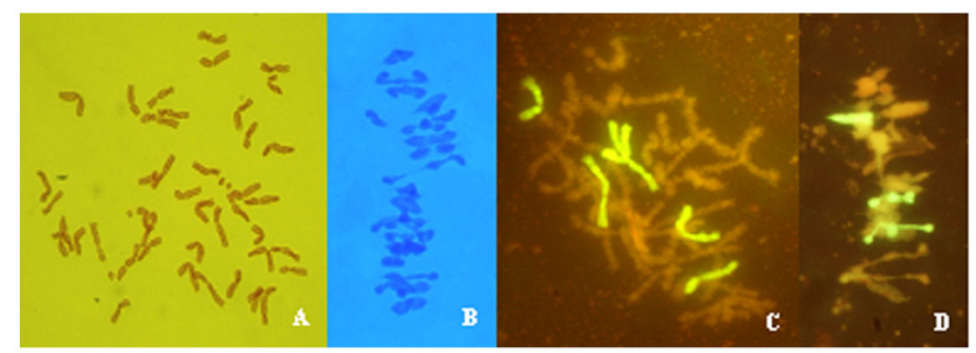

Figure 1. Mitotic and meiotic analyses of the substitution line 10DM50. A. Mitotic chromosomes from the root tip cells, $2 \mathrm{n}=42$; B. meiotic chromosomes at PMC metaphase I with 21 bivalents; C. mitotic GISH using the total DNA of Psathyrostachys huashanica as the probe, showing six chromosomes with hybridization fluorescing signals; D. meiotic GISH using the total DNA of Psathyrostachys huashanica as the probe, showing three bivalents with fluorescent signals. 
Mitotic and meiotic GISH, using the whole genomic DNA of $P$. huashanica $(2 \mathrm{n}=$ 14 , NsNs) as a probe, showed that 10DM50 had six chromosomes with clear hybridization signals in root tip cells (Figure 1C) and three bivalents with hybridization signals at PMC metaphase I (Figure 1D). This indicated that 10DM50 had six Ns chromosomes from $L$. mollis and that these six chromosomes paired to form three complete bivalents in the wheat background. Therefore, we deduced that 10DM50 was a genetically stable wheat-L. mollis alien substitution line with 36 chromosomes from wheat and three pairs of Ns genome chromosomes from $L$. mollis.

\section{SSR analysis}

The expected products of all of the SSR markers selected were obtained in octoploid Tritileymus M842-16 and wheat cv. 7182, which carried the entire D genome, whereas none of these products was obtained in durum wheat cv. D4286. In 10DM50, the expected products of the SSR markers specific to chromosomes $3 \mathrm{D}, 6 \mathrm{D}$, and $7 \mathrm{D}$ were not detected, whereas the expected products were obtained for the primers specific to chromosomes $1 \mathrm{D}, 2 \mathrm{D}, 4 \mathrm{D}$, and $5 \mathrm{D}$ (Table 1). Therefore, chromosomes $3 \mathrm{D}, 6 \mathrm{D}$, and $7 \mathrm{D}$ of wheat were absent in 10DM50.

\begin{tabular}{|c|c|c|c|c|c|c|}
\hline \multirow[t]{2}{*}{ Primer name and loci } & \multirow[t]{2}{*}{ Primer sequence } & \multirow[t]{2}{*}{$\operatorname{Tm}\left({ }^{\circ} \mathrm{C}\right)$} & \multicolumn{4}{|c|}{ PCR product } \\
\hline & & & 7182 & D4286 & 10DM50 & M842-16 \\
\hline Xgwm 458-1D & $\begin{array}{l}\text { F: AATGGCAATTGGAAGACATAGC } \\
\text { R: TTCGCAATGTTGATTTGGC }\end{array}$ & 60 & + & - & + & + \\
\hline Xgdm126-1D & $\begin{array}{l}\text { F: TCCATCATATCCGTAGCACA } \\
\text { R: CGTGGTTGATTTCAGGAGGT }\end{array}$ & 60 & + & - & + & + \\
\hline Xgwm484-2D & $\begin{array}{l}\text { F: ACATCGCTCTTCACAAACCC } \\
\text { R: AGTTCCGGTCATGGCTAGG }\end{array}$ & 55 & + & - & + & + \\
\hline Xgdm6-2D & $\begin{array}{l}\text { F: GATCAATCAAGCATGTGTGTGT } \\
\text { R: GGATGCCATGCCAAAGTATT }\end{array}$ & 55 & + & - & + & + \\
\hline$X g d m 72-3 \mathrm{D}$ & $\begin{array}{l}\text { F: TGGTTTTCTCGAGCATTCAA } \\
\text { R: TGCAACGATGAAGACCAGAA }\end{array}$ & 60 & + & - & - & + \\
\hline Xgdm128-3D & $\begin{array}{l}\text { F: TCACAGCAAACTCTGCGTG } \\
\text { R: ACAATTTGGCATGCTTCTCC }\end{array}$ & 60 & + & - & - & + \\
\hline Xgdm125-4D & $\begin{array}{l}\text { F: GCAGGCGTGTTACTCCAAGT } \\
\text { R: CCGAGGTGGATAGGAGGAAA }\end{array}$ & 60 & + & - & + & + \\
\hline Xgwm194-4D & $\begin{array}{l}\text { F: GATCTGCTCTACTCTCCTCC } \\
\text { R: CGACGCAGAACTTAAACAAG }\end{array}$ & 50 & + & - & + & + \\
\hline Xgdm153-5D & $\begin{array}{l}\text { F: TATAGGCAAATTAATTAAGACG } \\
\text { R: ATCTTTATGTGAGTACACTGC }\end{array}$ & 50 & + & - & + & + \\
\hline Xgwm212-5D & $\begin{array}{l}\text { F: AAGCAACATTTGCTGCAATG } \\
\text { R: TGCAGTTAACTTGTTGAAAGGA }\end{array}$ & 60 & + & - & + & + \\
\hline Xgdm98-6D & $\begin{array}{l}\text { F: CCATCCATGAAATGGCG } \\
\text { R: GCCCTTCACTAGCCTTCATG }\end{array}$ & 60 & + & - & - & + \\
\hline Xgdm132-6D & $\begin{array}{l}\text { F: ACCGCTCGGAGAAAATCC } \\
\text { R: AGGGGGGCAGAGGTAGG }\end{array}$ & 60 & + & - & - & + \\
\hline Xgwm295-7D & $\begin{array}{l}\text { F: GTGAAGCAGACCCACAACAC } \\
\text { R: GACGGCTGCGACGTAGAG }\end{array}$ & 60 & + & - & - & + \\
\hline Xgdm46-7D & $\begin{array}{l}\text { F: TGTGTTGGCCTTGTGGTG } \\
\text { R: CTACCCAATGCATCCCCTTA }\end{array}$ & 60 & + & - & - & + \\
\hline
\end{tabular}

Locus, sequences, and annealing temperatures (Tm) of the SSR primers. $(+)$ and (-) indicate whether products were present or absent, respectively. 


\section{EST-STS analysis}

EST-STS primer pairs that showed polymorphic bands between octoploid Tritileymus and common wheat 7182 were selected from 140 EST-STS primer pairs to detect the homology group attributes of the alien chromosomes in 10DM50. Three EST-STS primer pairs (BF291730, $B M 134465$, and $C D 373475$ ) for homoeologous group 3, three for homoeologous group 6 (BE426174, BE518349, and CD452568), and four for homoeologous group 7 (BQ170462, $B F 293421, B Q 161902$, and CD452422) produced specific bands in 10DM50, as well as in octoploid Tritileymus and L. mollis that contained the Ns chromosome; however, these bands were not produced in common wheat 7182 or Triticum durum D4286 (Table 2, Figures 2, 3, and 4). In contrast, the specific bands in octoploid Tritileymus and L. mollis produced by the EST-STS primer pairs for homoeologous groups 1, 2, 4, and 5, were not detected in 10DM50. These results indicated that substitution line 10DM50 might contain the homoeologous chromosomes of groups 3, 6, and 7 of Ns genome from L. mollis, i.e., $3 \mathrm{Ns}, 6 \mathrm{Ns}$, and $7 \mathrm{Ns}$.

\begin{tabular}{|c|c|c|c|c|}
\hline Mapped member accession No. & Chromosome location & Primer sequence & $\operatorname{Tm}\left({ }^{\circ} \mathrm{C}\right)$ & Product size (bp) \\
\hline$B F 291730$ & 3AL 3BL 3BS 3DL & $\begin{array}{l}\text { F: AGCAGCGCACGGTATTTACT } \\
\text { R: TTAAGAACCCAACCCACAGC }\end{array}$ & 60 & 1141 \\
\hline$B M 134465$ & 3AL 3BL 3DL & $\begin{array}{l}\text { F: CAATTGAACCCATCCGAAAG } \\
\text { R: CTGCCCGAACTATCCACAAT }\end{array}$ & 60 & 402 \\
\hline$C D 373475$ & 3AS 3BS 3DS & $\begin{array}{l}\text { F: CCGAAGACGAAGTCGAGAAC } \\
\text { R: ACACATCCCGTCCTTCTTTG }\end{array}$ & 60 & 463 \\
\hline BE426174 & 6DL C6A 6AS 6BL & $\begin{array}{l}\text { F: ATGAAGGTACGCCAAGACGA } \\
\text { R: GGTCGATTGCAAGCCATTAT }\end{array}$ & 60 & 962 \\
\hline BE518349 & 6AL 6BL 6DL & $\begin{array}{l}\text { F: GTGGACTTCGAGGAGCTGAC } \\
\text { R: CACTGCGCGATGATGTAGAG }\end{array}$ & 60 & 431 \\
\hline$C D 452568$ & 6AL 6BL 6DL & $\begin{array}{l}\text { F: TTTGCATTTTCGTCTGCAAG } \\
\text { R: TCGACACGAGCAAGATTCAC }\end{array}$ & 60 & 431 \\
\hline$B Q 170462$ & 7AS 7BS 7DS & $\begin{array}{l}\text { F: CTTCGTCCAGAAGGAACTGC } \\
\text { R: TGAAAGCAGCTCATGAGAAAG }\end{array}$ & 60 & 432 \\
\hline BF293421 & 7AL 7BL 7DL & $\begin{array}{l}\text { F: GTACCTCAAGGTCGGCAAGT } \\
\text { R: CTAGAACTCCTGGGAAGCGG }\end{array}$ & 60 & 407 \\
\hline BQ161902 & 7AL 7BL 7DL & $\begin{array}{l}\text { F: CTATACACAGGTTGGATGCCC } \\
\text { R: CAGTACTGCTAATTGGGCGG }\end{array}$ & 60 & 401 \\
\hline$C D 452422$ & 7AL 7BL 7DL & $\begin{array}{l}\text { F: GAAGTTCTTGAGCAGCTCCG } \\
\text { R: TCAGATGCCTACGATGATGG }\end{array}$ & 60 & 408 \\
\hline
\end{tabular}

\section{Evaluation of agronomic traits}

According to the analysis of variance (Table 3), 10DM50 showed a highly significant increase in spikes per plant and spike length, but different degrees of decrease in the number of spikelets per spike, and low self-fertility compared to all three of its parents. The plant height of 10DM50 was similar to that of durum wheat D4286, but lower than those of the common wheat parent 7182 and the octoploid Tritileymus M842. 10DM50 showed a slight increase in kernel number per spike compared to durum wheat D4286, but a significant decrease compared to octoploid Tritileymus M842 and common wheat 7182. The 1000-kernel-weight was higher in 10DM50 than in its common wheat parent 7182 and its octoploid parent M842-16, but lower than that of durum wheat D4286. Overall, the substitution line 10DM50 showed 
great improvements in plant height, spikes per plant, spike length, and 1000-kernel weight compared to its common wheat parent 7182 (Figure 5). The leaf rust resistance testing showed that 10DM50 and octoploid Tritileymus M842-16 had high resistance with type 0 infections, whereas the common wheat parent 7182 and the durum wheat parent D4286 were infected with type 4 and type 2, respectively. This indicated that 10DM50 had acquired the resistance gene(s) to leaf rust from octoploid Tritileymus M842-16.

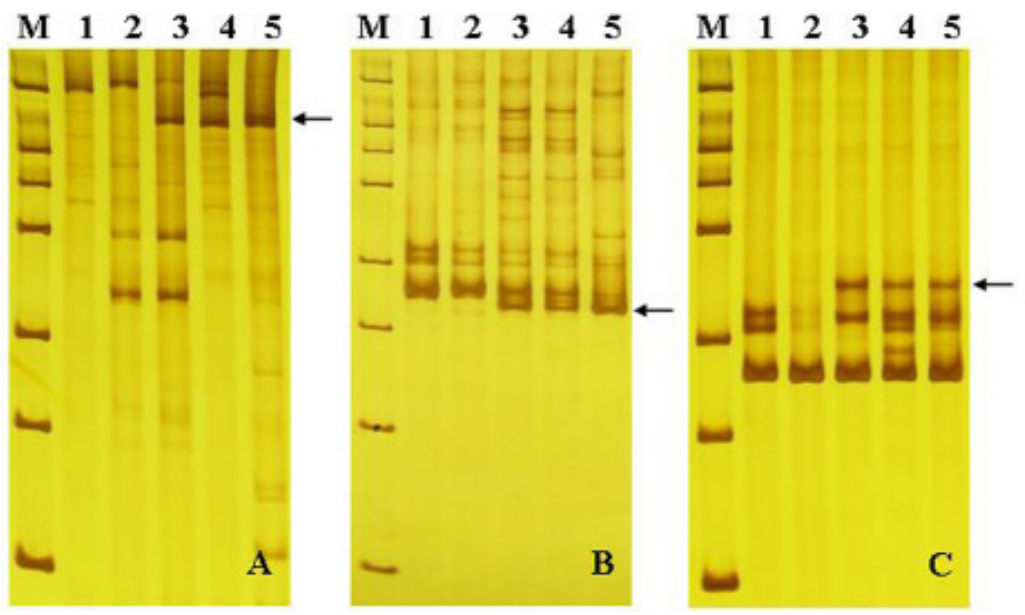

Figure 2. Identification of alien chromatin from Leymus mollis in substitution line 10DM50 using EST-STS primer pairs from group 3 of wheat. A. BF291730 specific primer for 3AL, 3B and 3DL; B. BM134465 for 3AL 3BL 3DL; C. $C D 373475$ for 3AS 3BS 3DS. Lane $M=$ marker; Lane $1=$ common wheat 7182 ; lane $2=$ durum wheat D4286; lane 3 = substitution line DM50; lane $4=$ octoploid Tritileymus M842; lane $5=$ Leymus mollis. Arrows show the target bands.

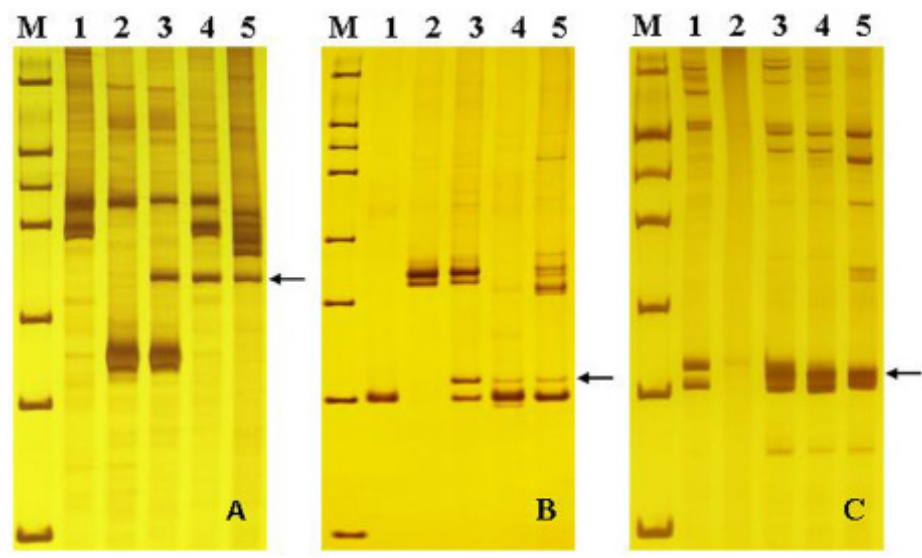

Figure 3. Identification of alien chromatin from Leymus mollis in substitution line 10DM50 using EST-STS primer pairs from group 6 of wheat. A. BE426174 specific primer for C6A, 6AS, 6BL and 6DL; B. BE518349 and C. CD452568 for 6AL, 6BL and 6DL. Lane $M=$ marker; Lane $1=$ common wheat 7182 ; lane $2=$ durum wheat D4286; lane 3 = substitution line DM50; lane 4 = octoploid Tritileymus M842; lane $5=$ Leymus mollis. Arrows show the target bands. 


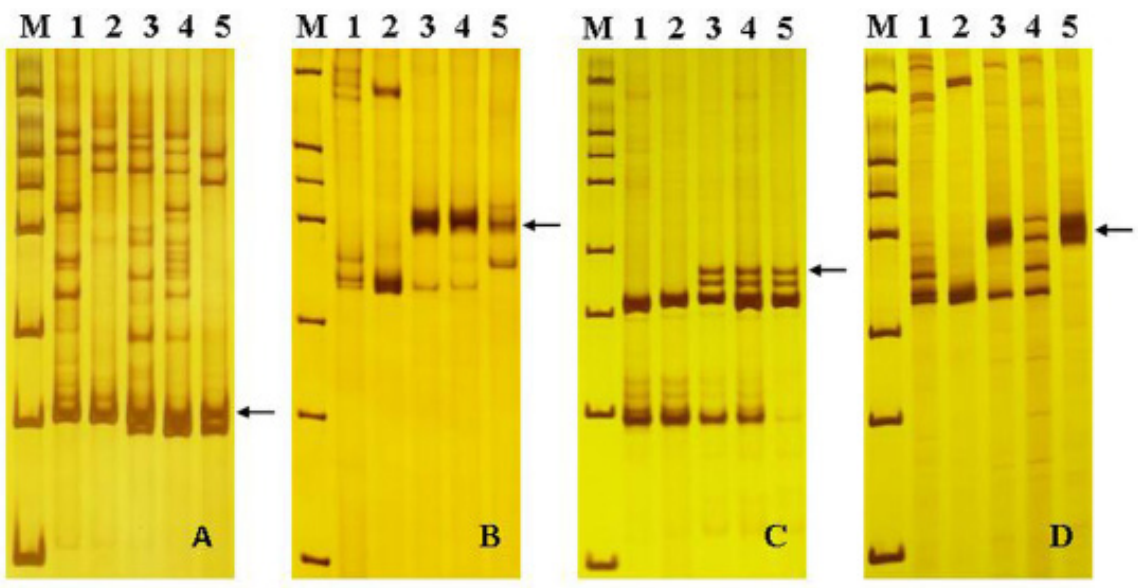

Figure 4. Identification of alien chromatin from Leymus mollis in substitution line 10DM50 using EST-STS primer pairs from group 7 of wheat. A. BQ170462 specific primer for 7AS, 7BS and 7DS; B. BF293421, C. BQ161902 and D. CD452422 for 7AL 7BL 7DL. Lane $M=$ marker; Lane $1=$ common wheat 7182; lane $2=$ durum wheat D4286; lane 3 = substitution line DM50; lane 4 = octoploid Tritileymus M842; lane $5=$ Leymus mollis. Arrows show the target bands.

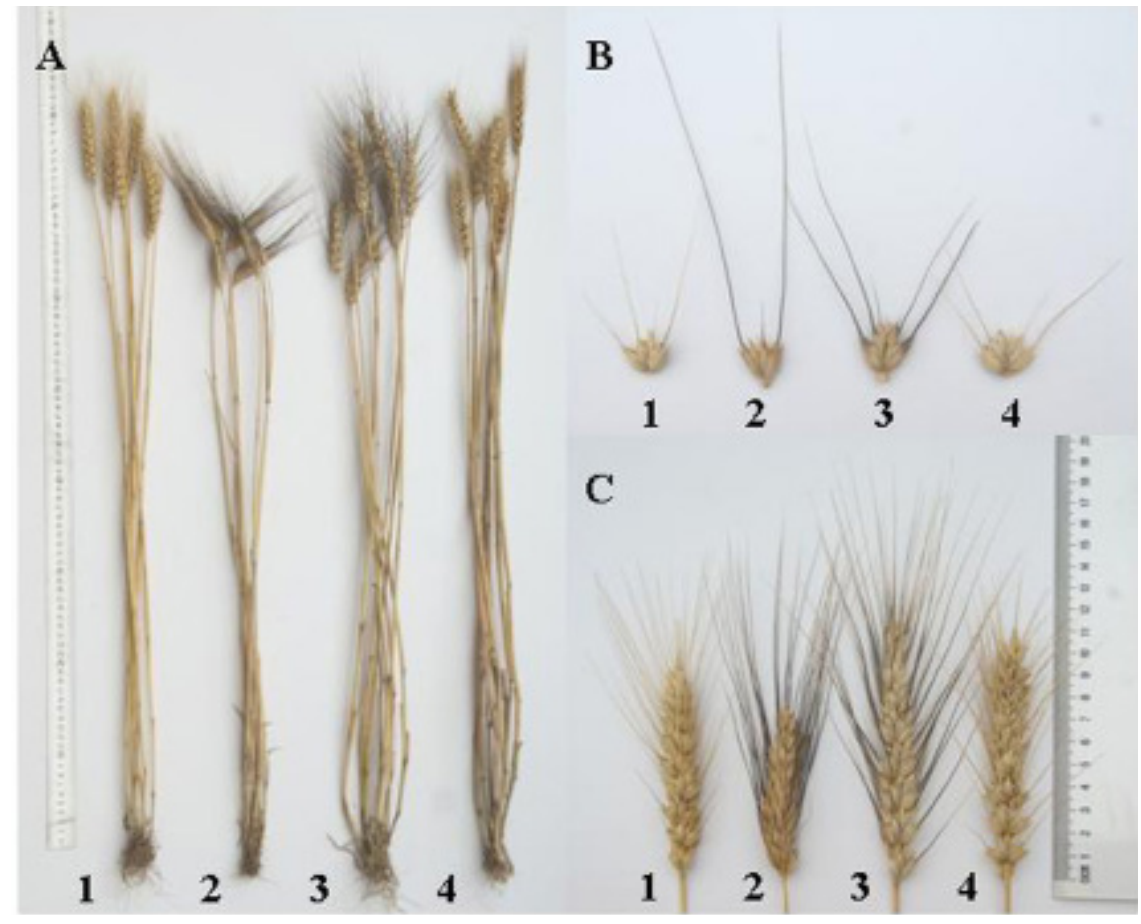

Figure 5. Morphological comparison of adult plants, spikes and spikelets for alien substitution line DM50 and its parents. A. Adult plants; B. spikes; C. spikelets. Lane 1= common wheat 7182; lane 2 = durum wheat D4286; lane 3 = substitution line DM50; lane 4 = octoploid Tritileymus M842. 


\begin{tabular}{|c|c|c|c|c|c|c|c|}
\hline Plant material & $\begin{array}{l}\text { Plant height } \\
(\mathrm{cm})\end{array}$ & $\begin{array}{l}\text { Spikes per } \\
\text { plant }\end{array}$ & $\begin{array}{l}\text { Spike length } \\
(\mathrm{cm})\end{array}$ & $\begin{array}{l}\text { Spikelets per } \\
\text { spike }\end{array}$ & $\begin{array}{l}\text { Kernels per } \\
\text { spike }\end{array}$ & $\begin{array}{c}\text { 1000-kernel } \\
\text { weight (g) }\end{array}$ & $\begin{array}{c}\text { Self-fertility } \\
(\%)\end{array}$ \\
\hline M842-16 & $88.40^{\mathrm{Aa}}$ & $8.40^{\mathrm{Bb}}$ & $9.78^{\mathrm{Bb}}$ & $19.64^{\mathrm{Aa}}$ & $52.68^{\mathrm{Aa}}$ & $42.14^{\mathrm{Cc}}$ & $58.08^{\mathrm{Cbc}}$ \\
\hline D4286 & $74.10^{\mathrm{Cc}}$ & $7.00^{\mathrm{Bb}}$ & $6.77^{\mathrm{Dd}}$ & $18.20^{\mathrm{Bbc}}$ & $39.32^{\mathrm{Bb}}$ & $49.64^{\mathrm{Aa}}$ & $68.03^{\mathrm{ABb}}$ \\
\hline 7182 & $83.48^{\mathrm{Bb}}$ & $6.20^{\mathrm{Bb}}$ & $8.62^{\mathrm{Cc}}$ & $18.88^{\mathrm{ABab}}$ & $49.32^{\mathrm{ABa}}$ & $36.86^{\mathrm{Dd}}$ & $81.33^{\mathrm{Aa}}$ \\
\hline 10DM50 & $75.16^{\mathrm{Cc}}$ & $12.40^{\mathrm{Aa}}$ & $11.58^{\mathrm{Aa}}$ & $17.92^{\mathrm{Bc}}$ & $40.64^{\mathrm{Bb}}$ & $44.19^{\mathrm{Bb}}$ & $55.84^{\mathrm{Cc}}$ \\
\hline
\end{tabular}

Capital and small letters mean the significant differences at the levels of $\mathrm{P}<0.01$ and $\mathrm{P}<0.05$, respectively.

\section{DISCUSSION}

The cytogenetic stability of alien substitution lines, addition lines, and translocation lines is of theoretical and practical significance for their application in genetics and plant breeding. The maintenance of such lines requires regular meiotic pairing and recombination in the subsequent selfing progenies (Cifuentes and Benavente, 2009). According to the chromosome pairing analyzed in this study, the line $10 \mathrm{DM} 50$ with $2 \mathrm{n}=42$ showed regular meiosis, indicating that 10DM50 was cytogenetically stable.

GISH is one of the most versatile and powerful research tools currently used in plant chromosome and genome research. It has been used for characterization of genomes and chromosomes in hybrid polyploids, hybrid plants, partial allopolyploids, polyhaploids, and recombinant breeding lines, as well as for the localization and detection of the amount of introgressed alien chromatin (Raina and Rani, 2001). However, in the present study, it was difficult to define the alien chromosomes in DM50 by GISH using the DNA of L. mollis as a probe due to the allotetraploidy of L. mollis (NsNsXmXm, $2 \mathrm{n}=28$ ) with the Ns genome originating from the genus Psathyrostachys and the Xm genome of unknown origin. Therefore, the total DNA of $P$. huashanica (NsNs, $2 \mathrm{n}=14$ ) was used as a probe to determine the alien chromosomes in 10DM50 during mitotic and meiotic GISH, and the results showed that 10DM50 was a cytogenetically stable wheat-L. mollis substitution line with three pairs of Ns chromosomes.

Using DNA markers to identify the alien chromosomes has enabled great progress in plant chromosome engineering breeding research, and its potential is far from exhausted. This method is particularly important for the genetic analysis of alien chromatin from wheat relatives that lack specifically developed DNA markers (Dobrovolskaya et al., 2009). Because genomes A and B from octoploid Tritileymus should match well with genomes A and B of durum wheat, these should be inherited stably in hybrids of octoploid Tritileymus M842-16 x Triticum durum D4286, and substitutions are instead most likely to occur between genomes D and Ns from octoploid Tritileymus. Therefore, SSR markers from wheat genome D were used to identify the missing chromosomes, and the results showed that chromosomes $3 \mathrm{D}, 6 \mathrm{D}$, and 7D were absent from line 10DM50.

Comparative genome mapping across cereal species has shown that homoeologous chromosomes are generally similar and conserved in terms of their gene content and gene order (Gale and Devos, 1998). GISH and SSR analyses of the substitution line 10DM50 revealed three pairs of Ns chromosomes from L. mollis and that the chromosomes $3 \mathrm{D}, 6 \mathrm{D}$, and $7 \mathrm{D}$ of wheat were absent. We deduced that the substitution most likely occurred between homoeologous chromosomes from the $\mathrm{D}$ and Ns genomes, that is, chromosomes $3 \mathrm{D}, 6 \mathrm{D}$, and 7D of wheat were replaced by chromosomes $3 \mathrm{Ns}, 6 \mathrm{Ns}$, and $7 \mathrm{Ns}$ from L. mollis, respectively. 
In order to support this deduction, EST-STS primer pairs, selected from different chromosome groups of wheat that showed polymorphic bands between common wheat 7182 and octoploid Tritileymus M842, were employed to identify the alien chromosomes in DM50. Indeed, the results showed that the EST-STS primer pairs of group 3, group 6, and group 7 detected special bands in the substitution line 10DM50. The bands were also detected in octoploid Tritileymus M842 and L. mollis, which both contain the Ns genome. This suggested that the three pairs of alien chromosomes in 10DM50 were most likely transferred from group 3, group 6, and group 7, i.e. $3 \mathrm{Ns}, 6 \mathrm{Ns}$, and $7 \mathrm{Ns}$ from L. mollis. The specific EST-STS primer pairs could be used to develop special markers for detecting Ns chromatin in wheat- $L$. mollis progenies, which should facilitate the detection of $L$. mollis chromatin in a wheat background.

10DM50, a multiple alien substitution line from octoploid Tritileymus M842-16 x Triticum durum cv. D4286, with 36 chromosomes from wheat and three pairs of Ns chromosomes from L. mollis, was selected and identified using cytogenetic and molecular methods. It was shown to possess many valuable traits that could be transferred into common wheat in breeding programs, such as low plant height, high number of spikes, long spikes, high 1000-kernel-weight, and high resistance to leaf rust. It could also be used as a precious intermediate material to develop translocation lines, and eventually to transfer useful gene(s) from L. mollis to common wheat successfully.

\section{ACKNOWLEDGMENTS}

Research supported by the National High Technology Research and Development Program of China (\#2011AA100501), the Major Innovation Project for Science and Technology of Shaanxi Province (\#2011KTZB02-01), and the Tang Zhong-Ying Breeding Funding Project at the Northwest A\&F University, which are greatly appreciated.

\section{REFERENCES}

Anamthawat-Jónsson K, Bödvarsdóttir SK, Bragason BT, Gudmundsson J, et al. (1997). Wide hybridization between wheat (Triticum L.) and lymegrass (Leymus Hochst.). Euphytica 93: 293-300.

Baum M, Lagudah ES and Appels R (1992). Wide crosses in cereals. Annu. Rev. Plant Physiol. Plant Mol. Biol. 43: $117-$ 143.

Chen SY, Fu J and Gao LZ (1985). The hybridization between Triticum aestivum and Leymus mollis. Acta Bot. Bor.-Occ. Sinica 5: 260-266.

Cifuentes M and Benavente E (2009). Wheat-alien metaphase I pairing of individual wheat genomes and D genome chromosomes in interspecific hybrids between Triticum aestivum L. and Aegilops geniculata Roth. Theor. Appl. Genet. 119: 805-813.

Cota-Sánchez JH, Remarchuk K and Ubayasena K (2006). Ready-to-use DNA extracted with a CTAB method adapted for herbarium specimens and mucilaginous plant tissue. Plant Mol. Biol. Rep. 24: 161-167.

Dobrovolskaya OB, Sourdille P, Bernard M and Salina EA (2009). Chromosome synteny of the a genome of two evolutionary wheat lines. Russ. J. Genet. 45: 1368-1375.

Fu J, Chen SY and Zhang AJ (1993). Studies of the formation and cytogenetics of octoploid Tritileymus. Acta Genet. Sin. 20: $317-323$.

Fu J, Chen S, Zhang A, Hou W, et al. (1996). Cytogenetic studies on the cross progenies between octoploid Tritileymus and Triticum aestivum. Acta Genet. Sin. 23: 24-31.

Gale MD and Devos KM (1998). Plant comparative genetics after 10 years. Science 282: 656-659.

Jiang J, Friebe B and Gill BS (1994). Recent advances in alien gene transfer in wheat. Euphytica 73: 199-212.

Jing JX, Guo P, Li LY, Fu J, et al. (2001). Resistance of Elymus mollis (Trin) Hara. to Puccinia striiformus f. sp. tritici. J. Northwest Sci-Tech Univ. Agric. Forestry 29: 107-110. 
Kishii M, Wang RR and Tsujimoto H (2003). Characteristics and behaviour of the chromosomes of Leymus mollis and $L$. racemosus (Triticeae, Poaceae) during mitosis and meiosis. Chromosome Res. 11: 741-748.

Kishii M, Yamada T, Sasakuma T and Tsujimoto H (2004). Production of wheat-Leymus racemosus chromosome addition lines. Theor. Appl. Genet. 109: 255-260.

Lin ZS, Cui ZF, Zeng XY, Ma YZ, et al. (2007). Analysis of wheat-Thinopyrum intermedium derivatives with BYDVresistance. Euphytica 158: 109-118.

Merker A and Lantai K (1998). Hybrids between wheats and perennial Leymus and Thinopyrum species. Acta Agric. Scand. Section B - Soil \& Plant 47: 48-51.

Pestsova E, Ganal MW and Roder MS (2000). Isolation and mapping of microsatellite markers specific for the D genome of bread wheat. Genome 43: 689-697.

Raina SN and Rani V (2001). GISH technology in plant genome research. Methods Cell Sci. 23: 83-104.

Röder MS, Korzun V, Wendehake K, Plaschke J, et al. (1998). A microsatellite map of wheat. Genetics 149: 2007-2023.

Tsitsin N (1965). Remote hybridisation as a method of creating new species and varieties of plants. Euphytica 14: 326-330.

Walling JG, Pires JC and Jackson SA (2005). Preparation of samples for comparative studies of plant chromosomes using in situ hybridization methods. Methods Enzymol. 395: 443-460.

Wang XP, Fu J, Zhang XQ, Jing JK, et al. (2000). Molecular cytogenetic study on genome constitutions of octoploid Tritileymus. Acta Bot. Sin. 42: 586.

Wang RR and Jensen KB (1994). Absence of the J genome in Leymus species (Poaceae: Triticeae): evidence from DNA hybridization and meiotic pairing. Genome 37: 231-235.

Zhao JX, Du WL, Wu J, Cheng XN, et al. (2012). Development and identification of a wheat-Leymus mollis multiple alien substitution line. DOI: 10.1007/s10681-012-0772-3. Euphytica.

Zhou XC, Yang XM, Li XQ and Li LH (2010). Genome origins in Leymus (Poaceae: Triticeae): evidence of maternal and paternal progenitors and implications for reticulate evolution. Plant Syst. Evol. 289: 165-179. 